\title{
Response of serum 25-hydroxyvitamin D concentrations to vitamin D supplementation during lactation
}

\author{
J. Y. Zhang ${ }^{1}$, A. Lucey ${ }^{1}$, J. R. Higgins ${ }^{2}$, K. D. Cashman ${ }^{1}$ and M. Kiely ${ }^{1}$ \\ ${ }^{1}$ Vitamin D Research Group, School of Food and Nutritional Sciences, University College Cork, Republic of Ireland and \\ ${ }^{2}$ Anu Research Centre, Department of Obstetrics and Gynaecology, Cork University Maternity Hospital, University College \\ Cork, Ireland
}

Pregnancy, lactation and early childhood are life stages when the risk of low vitamin D status is high and the knowledge basis for determining nutritional requirements for vitamin $\mathrm{D}$ is weak. The current dietary reference intervals (DRI) for vitamin D in pregnant and lactating women are the same as those in non-pregnant adult females below 70 years $(600 \mathrm{IU} / 15 \mu \mathrm{g} / \mathrm{d})^{(1)}$. The aim of the current study was to investigate vitamin $\mathrm{D}$ requirements during lactation. We conducted a double-blind randomised placebo-controlled trial across three intervention groups using $20 \mu \mathrm{g} / \mathrm{d}$ of vitamin $\mathrm{D}_{3}$ (to achieve a total vitamin $\mathrm{D}$ intake of $\sim 25 \mu \mathrm{g} / \mathrm{d}$ ), with or without $500 \mathrm{mg}$ Ca, or placebo, over 12 weeks of lactation. The study protocol was implemented across a calendar year to account for seasonal effects.

Concentrations of serum 25-hydroxyvitamin $\mathrm{D}(\mathrm{s} 25(\mathrm{OH}) \mathrm{D})$ were measured at baseline $(\mathrm{BL})$ and endpoint $(\mathrm{EP})$ in mothers and in umbilical cord blood using ELISA. Vitamin D metabolites $\left(\mathrm{D}_{3}, \mathrm{D}_{2}\right.$ and $\left.25(\mathrm{OH}) \mathrm{D}\right)$ were quantified in expressed breast milk at four time points during the intervention study using HPLC. Dietary intakes of vitamin D and Ca, anthropometric data, socio-demographic and lifestyle data were collected, as well as antenatal supplement use and habitual sunshine exposure. The s25(OH)D data are described here.

\begin{tabular}{lccccc}
\hline $25(\mathrm{OH}) \mathrm{D}(\mathrm{nmol} / \mathrm{L})$ & Total $(n$ 100) & Placebo $(n$ 27) & Vitamin D $(n$ 36) & D and Ca $(n$ 37) & $P$ \\
\hline Baseline* & $43.4(31.5-60.1)$ & $45.2(29.2-64.9)$ & $45.4(38.1-54.2)$ & $39.5(30.1-62.6)$ & 0.835 \\
Endpoint $\dagger$ & $71.8(58.6-88.7)$ & $48.3(36.7-65.6)$ & $78.4(67.9-94.1)$ & $75.9(67.7-98.0)$ & $<0.001$ \\
\hline Median; interquartile range in parentheses; *One-way ANOVA followed by Tukey's test. \\
†ANCOVA adjusting for BL s25(OH)D, dietary intake of vitamin D and season of EP blood sampling.
\end{tabular}

At BL, 21 and $63 \%$ had a s25(OH)D level below the thresholds for vitamin D deficiency and sufficiency of 30 and $50 \mathrm{nmol} / \mathrm{l}$, respectively. Season of blood sampling was the main determinant of BL s25(OH)D (adj. $\left.R^{2}=0.338 ; \beta=0.571 ; P<0.001\right)$, as expected. Other determinants were parity and total vitamin $\mathrm{D}$ intake $(\beta=0.157$ and 0.150 , respectively; $P<0.05)$. Mean (SD) cord s25(OH)D levels were $33.8(14.8) \mathrm{nmol} / 1(n 92)$ and were $78 \%$ of maternal levels on average $\left(R^{2}=0.7 ; P<0.001\right)$. Season of birth $\left(\operatorname{adj} . R^{2}=0.274 ; \beta=0.137\right)$ and antenatal vitamin D supplement use $\left(\operatorname{adj} . R^{2}=0.004 ; \beta=0.140\right)$ were independent predictors of cord s $25(\mathrm{OH}) \mathrm{D}$ levels $($ both $P<0.05)$.

A final sample of 100 women completed the intervention protocol, of which ninety were more than $80 \%$ compliant. The intervention considerably increased s25(OH)D levels in the treatment groups by $\sim 30 \mathrm{nmol} / \mathrm{l}$ (see Table) with no difference in the EP concentrations between women who received vitamin $\mathrm{D}$ only and those who received vitamin $\mathrm{D}+\mathrm{Ca}$. Given that the average habitual vitamin $\mathrm{D}$ intake in the group was $4.3 \mu \mathrm{g} / \mathrm{d}$, supplementation with $20 \mu \mathrm{g} / \mathrm{d}$ vitamin $\mathrm{D}_{3}$ to achieve a total intake of $\sim 25 \mu \mathrm{g} / \mathrm{d}$, maintained s25(OH)D levels $>30 \mathrm{nmol} / \mathrm{l}$ in all lactating women and brought $96 \%$ above the desirable threshold of $50 \mathrm{nmol} / \mathrm{l}$.

In conclusion, the current DRI of $15 \mu \mathrm{g} / \mathrm{d}$ is inadequate to achieve a target s25(OH)D of $50 \mathrm{nmol} / 1$ in $97.5 \%$ of lactating women at a latitude of $51^{\circ} \mathrm{N}$.

Funded by the Irish Department of Agriculture, Fisheries and Food through the Food Institutional Research Measure.

1. IOM (Institute of Medicine) (2011) Dietary Reference Intakes for Calcium and Vitamin D. Washington, DC: The National Academies Press. 